\title{
GENERALIZATION OF THE SCHMIDT LEMMA TO THE CASE OF $n$-NORMAL AND $d$-NORMAL OPERATORS IN A BANACH SPACE
}

\author{
V. F. Zhuravlev
}

UDC 517.983

\begin{abstract}
We generalize the known Schmidt lemma to the case of linear, bounded, normally solvable operators that are $n$-normal or $d$-normal in infinite-dimensional Banach spaces. It is assumed that the kernels and images of these operators have complements in these spaces.
\end{abstract}

The Schmidt lemma [1] is most completely studied and widely used for the generalized inversion of linear, bounded, normally solvable Fredholm operators (with nonzero kernels) in the form of the so-called Schmidt construction [2]. Its analog for Noetherian operators in finite-dimensional Banach and Hilbert spaces was considered in [3].

The aim of the present paper is to prove statements that generalize the Schmidt lemma to the case of bounded normally extendable operators that are $n$-normal or $d$-normal and act in infinite-dimensional Banach spaces.

\section{Statement of the Problem}

Let $L$ be a linear, bounded, normally solvable operator that acts from a Banach space $\mathbf{B}_{1}$ into a Banach space $\mathbf{B}_{2}$. Denote the dimensions of the null spaces of the operator $L$ and its adjoint $L^{*}$ by $\operatorname{dim} N(L)=\mu$ and $\operatorname{dim} N\left(L^{*}\right)=v$, respectively. According to S. Krein's classification [4], a normally solvable operator $L$ is $n$-normal if $\mu$ is finite and $v$ is infinite, and it is $d$-normal if $\mu$ is infinite and $v$ is finite.

If $L: \mathbf{B}_{1} \rightarrow \mathbf{B}_{2}$ is a linear bounded $n$-normal operator, then we assume that its image $R(L)$ has a complement in the space $\mathbf{B}_{2}$ [5], i.e.,

$$
B_{2}=Y \oplus R(L),
$$

and if $L: \mathbf{B}_{1} \rightarrow \mathbf{B}_{2}$ is a linear bounded $d$-normal operator, then its kernel $N(L)$ has a complement in the space $\mathbf{B}_{1}$, i.e.,

$$
B_{1}=N(L) \oplus X
$$

\section{Main Result}

First, we consider $n$-normal operators. By virtue of its finite dimensionality $(\mu<\infty)$, the subspace $N(L)$ has a complete system of basis elements $\left\{f_{i}\right\}_{i=1}^{\mu} \subset N(L), f_{i}=\operatorname{col}\left(f_{i}^{(1)}, f_{i}^{(2)}, f_{i}^{(3)}, \ldots\right)$. Assume that the space $\mathbf{B}_{2}$ has a basis. It is known [6, p. 131] that $\mathbf{B}_{2}^{*}$ also has a basis. Therefore, the subspace $N^{*}(L) \subset \mathbf{B}_{2}^{*}$ has a complete system of basis elements (functionals) $\left\{\varphi_{s}(\cdot)\right\}_{s=1}^{\infty} \subset N\left(L^{*}\right), \varphi_{s}(\cdot)=\operatorname{col}\left(\varphi_{s}^{(1)}(\cdot), \varphi_{s}^{(2)}(\cdot), \varphi_{s}^{(3)}(\cdot), \ldots\right)$. For the elements $\left\{f_{i}\right\}_{i=1}^{\mu}$ and functionals $\left\{\varphi_{s}(\cdot)\right\}_{s=1}^{\infty}$, there exist an adjoint biorthogonal [7] system of functionals $\left\{\gamma_{j}(\cdot)\right\}_{j=1}^{\mu} \subset \mathbf{B}_{1}^{*}, \gamma_{j}(\cdot)=\operatorname{col}\left(\gamma_{j}^{(1)}(\cdot), \gamma_{j}^{(2)}(\cdot), \gamma_{j}^{(3)}(\cdot), \ldots\right)$, and an adjoint biorthogonal complete system of

Zhitomir National Agroecological University, Zhitomir, Ukraine; e-mail: vfz2008@ukr.net.

Translated from Neliniini Kolyvannya, Vol. 12, No. 4, pp. 443-450, October-December, 2009. Original article submitted April 6, 2009. 
elements $\left\{\psi_{k}\right\}_{k=1}^{\infty} \subset \mathbf{B}_{2}, \psi_{k}=\operatorname{col}\left(\psi_{k}^{(1)}, \psi_{k}^{(2)}, \psi_{k}^{(3)}, \ldots\right)$. Note that, according to the Hahn-Banach theorem, each functional $\left\{\gamma_{j}(\cdot)\right\}_{j=1}^{\mu}$ defined on the subspace $N(L) \subset \mathbf{B}_{1}$ can be extended, with preservation of norm, to the entire space $\mathbf{B}_{1}$.

Let

$$
\begin{gathered}
X=\left(f_{1}, f_{2}, \ldots, f_{\mu}\right), \quad \Gamma(\cdot)=\left(\gamma_{1}(\cdot), \gamma_{2}(\cdot), \ldots, \gamma_{\mu}(\cdot)\right)^{T} \\
\Phi(\cdot)=\left(\varphi_{1}(\cdot), \varphi_{2}(\cdot), \ldots, \varphi_{k}(\cdot), \ldots\right)^{T}, \quad \Psi=\left(\psi_{1}, \psi_{2}, \ldots, \psi_{k}, \ldots\right)
\end{gathered}
$$

denote, respectively, $\infty \times \mu, \mu \times \infty, \infty \times \infty$, and $\infty \times \infty$ matrices; furthermore, $\Gamma(X)=E_{\mu}$ and $\Phi(\Psi)=E_{\infty}$, where $E_{\mu}$ and $E_{\infty}$ are the identity matrices.

We construct a projection operator $\mathcal{P}_{N(L)}: \mathbf{B}_{1} \rightarrow N(L)$ according to the formula

$$
\mathcal{P}_{N(L)}(\cdot)=X \Gamma(\cdot), \quad \mathcal{P}_{N(L)}: B_{1} \rightarrow B_{1}
$$

To construct a projection operator $\mathcal{P}_{Y}: B_{2} \rightarrow B_{2}$, we define the sequence of projectors

$$
\mathcal{P}_{Y^{(j)}}(\cdot)=\Psi_{j} \Phi_{j}(\cdot)
$$

of the space $\mathbf{B}_{2}$ to the subspaces $Y_{j} \subset Y$ spanned by the elements $\left\{\psi_{k}\right\}_{k=1}^{j}$.

Lemma 1. The sequence (4) of projectors $\mathcal{P}_{Y^{(j)}}$ converges strongly (pointwise) to the projector

$$
\mathcal{P}_{Y}(\cdot)=\Psi \Phi(\cdot)=\lim _{j \rightarrow \infty} \Psi_{j} \Phi_{j}(\cdot), \quad \mathcal{P}_{Y}: B_{2} \rightarrow Y,
$$

where $Y \subset \mathbf{B}_{2}$ is an infinite-dimensional space spanned by the complete system of elements $\left\{\psi_{s}\right\}_{s=1}^{\infty}$.

Proof. According to the definition of strong convergence in the norm of the space $\mathbf{B}_{2}$, with regard for the definition of the matrices $\Phi$ and $\Psi$ we get

$$
\begin{aligned}
\left\|\mathcal{P}_{Y} y-\mathcal{P}_{Y_{j}} y\right\| & =\left\|\sum_{\xi=1}^{\infty} \varphi_{\xi}(y) \psi_{\xi}-\sum_{\xi=1}^{j} \varphi_{\xi}(y) \psi_{\xi}\right\| \\
& =\left\|\sum_{\xi=j+1}^{\infty} \varphi_{\xi}(y) \psi_{\xi}\right\| \leq \sum_{\xi=j+1}^{\infty}\left\|\varphi_{\xi}(y) \psi_{\xi}\right\| \quad \forall y \in Y \subset \mathbf{B}_{2} .
\end{aligned}
$$

The quantity

$$
\sum_{\xi=j+1}^{\infty}\left\|\varphi_{\xi}(y) \psi_{\xi}\right\|
$$

tends to zero as $j \rightarrow \infty$ as a remainder of the expansion

$$
\sum_{\xi=1}^{\infty} \varphi_{\xi}(y) \psi_{\xi}
$$


of an element $y \in Y$ in the system of elements $\left\{\psi_{\xi}\right\}_{\xi=1}^{\infty}$. Since the functionals $\left\{\varphi_{j}(\cdot)\right\}_{j=1}^{\infty}$ can be extended to the entire space $\mathbf{B}_{2}$ with preservation of norm, we can conclude that

$$
\sum_{\xi=j+1}^{\infty}\left\|\varphi_{\xi}(y) \psi_{\xi}\right\| \rightarrow 0 \quad \text { as } \quad j \rightarrow \infty
$$

for any $y \in \mathbf{B}_{2}$.

The lemma is proved.

Let us show that the constructed projectors divide the spaces $\mathbf{B}_{1}$ and $\mathbf{B}_{2}$ into mutually complementary subspaces according to relations (1) and (2).

Lemma 2. The operators $\mathcal{P}_{N(L)}$ and $\mathcal{P}_{Y}$ are bounded projectors in the Banach spaces $\mathbf{B}_{1}$ and $\mathbf{B}_{2}$ and divide these spaces into direct sums of closed subspaces according to relations (1) and (2).

Proof. First, we prove that the operators $\mathcal{P}_{N(L)}$ and $\mathcal{P}_{Y}$ are projectors, i.e., that they satisfy the conditions $\mathcal{P}_{N(L)}^{2}=\mathcal{P}_{N(L)}$ and $\mathcal{P}_{Y}^{2}=\mathcal{P}_{Y}$. Indeed, we have

$$
\mathcal{P}_{N(L)}^{2}(\cdot)=\mathcal{P}_{N(L)}\left(\mathcal{P}_{N(L)}(\cdot)\right)=X \Gamma(X \Gamma(\cdot))=X \Gamma(X) \Gamma(\cdot)=X \Gamma(\cdot)=\mathcal{P}_{N(L)}(\cdot)
$$

because $\Gamma(X)=E_{\mu}$, and

$$
\mathcal{P}_{Y}^{2}(\cdot)=\mathcal{P}_{Y}\left(\mathcal{P}_{Y}(\cdot)\right)=\Psi \Phi(\Psi \Phi(\cdot))=\Psi \Phi(\Psi) \Phi(\cdot)=\Psi \Phi(y \cdot)=\mathcal{P}_{Y}(\cdot)
$$

because $\Phi(\Psi)=E_{\nu}$.

Thus, the projectors $\mathcal{P}_{N(L)}$ and $\mathcal{P}_{Y}$ divide the spaces $\mathbf{B}_{1}$ and $\mathbf{B}_{2}$ into direct topological sums of closed subspaces:

$$
\mathbf{B}_{1}=N\left(\mathcal{P}_{N(L)}\right) \oplus R\left(\mathcal{P}_{N(L)}\right), \quad \mathbf{B}_{2}=N\left(\mathcal{P}_{Y}\right) \oplus R\left(\mathcal{P}_{Y}\right)
$$

Further, we show that

$$
\begin{gathered}
N(L)=R\left(\mathcal{P}_{N(L)}\right), \quad R(L)=N\left(\mathcal{P}_{Y}\right), \\
Y=R\left(\mathcal{P}_{Y}\right), \quad X=N\left(\mathcal{P}_{N(L)}\right) .
\end{gathered}
$$

Since $L \mathcal{P}_{N(L)} x=L X \Gamma(x)=0, x \in \mathbf{B}_{1}$, we have $R\left(\mathcal{P}_{N(L)}\right) \subset N(L)$. Let $x \in N(L)$. Then $x=X c$. Applying the matrix of functionals $\Gamma$ to the last equality, we get $c=\Gamma(x)$, i.e., $x=X \Gamma(x)$. Therefore, $x=\mathcal{P}_{N(L)} x$ and $x \in R\left(\mathcal{P}_{N(L)}\right)$. Thus, $N(L) \subset R\left(\mathcal{P}_{N(L)}\right)$, and the first equality in (5) is proved.

Since $\mathcal{P}_{Y} L x=\Psi \Phi(L z)=\Psi\left(L^{*} \Phi\right)(z)=0 \quad\left(\varphi_{S}\right.$ are basis vectors of the null space of the operator $\left.L^{*}\right)$, we have $R(L) \subset N\left(\mathcal{P}_{Y}\right)$. On the other hand, if $y \in N\left(\mathcal{P}_{Y}\right)$, then

$$
\mathcal{P}_{Y} y=\Psi \Phi(y)=0,
$$

i.e., $\varphi_{s}(y)=0, s=1,2, \ldots, \infty$. By virtue of the normal solvability of the operator $L$, this means that $y \in$ $R(L)$. Therefore, $N\left(\mathcal{P}_{Y}\right) \subset R(L)$, and the proof of the second equality in (5) is completed. 
The third and the fourth equality in (5) are proved by analogy.

Thus, the projectors $\mathcal{P}_{N(L)}$ and $\mathcal{P}_{Y}$ divide the Banach spaces $B_{1}$ and $B_{2}$ into direct sums of closed subspaces according to relations (1) and (2).

The boundedness of the projector $\mathcal{P}_{N(L)}$ follows from its finite dimensionality, and the boundedness of the projector $\mathcal{P}_{Y}$ follows from the complementability of the image $R(L)$ of the operator $L$ [8].

The lemma is proved.

Since the system of basis elements $\left\{\varphi(\cdot)_{s}\right\}_{s=1}^{\nu} \subset B_{2}^{*}$ of the null space $N\left(L^{*}\right)$ and the system of elements $\left\{\psi_{s}\right\}_{s=1}^{v} \subset Y \subset B_{2}$ are adjoint biorthogonal, $\varphi_{s}\left(\psi_{k}\right)=\delta_{s k}$, there exists a one-to-one correspondence between them. Therefore, the subspaces $N\left(L^{*}\right)$ and $Y$ are isomorphic and have the same dimension: $\operatorname{dim} N\left(L^{*}\right)=$ $\operatorname{dim} Y$. Since $\mu$ is finite and $v$ is infinite, we can establish an isomorphism between $N(L)$ and a certain subspace $Y_{1} \subset Y$.

We now construct this isomorphism.

Let

$$
\bar{\Phi}(\cdot)=\left(\bar{\varphi}_{1}(\cdot), \bar{\varphi}_{2}(\cdot), \ldots, \bar{\varphi}_{\mu}(\cdot)\right)^{T} \quad \text { and } \quad \bar{\Psi}=\left(\bar{\psi}_{1}, \bar{\psi}_{2}, \ldots, \bar{\psi}_{\mu}\right)
$$

denote, respectively, $\mu \times \infty$ and $\infty \times \mu$ matrices composed of $\mu$ rows and columns of the matrices $\Phi$ and $\Psi$, respectively. The matrix $\bar{\Psi}$ is composed of the system of elements $\left\{\bar{\psi}_{k}\right\}_{k=1}^{v} \subset\left\{\psi_{k}\right\}_{k=1}^{\infty}$ spanning the subspace $Y_{1}$. The matrix $\bar{\Phi}$ is composed of functionals $\left\{\bar{\varphi}_{s}\right\}_{s=1}^{v} \subset\left\{\varphi_{s}\right\}_{s=1}^{\infty}$ that satisfy the relation $\bar{\Phi}(\bar{\Psi})=E_{\mu}$. We construct a linear, bounded, invertible operator $J: N(L) \rightarrow Y_{1} \subseteq Y$ that performs an isomorphism of $N(L)$ onto $Y_{1}$ and its inverse $J^{-1}: Y_{1} \rightarrow N(L)$ according to the relations

$$
\begin{aligned}
& J(\cdot)=\bar{\Psi} \Gamma(\cdot), \quad(\cdot) \in N(L), \\
& J^{-1}(\cdot)=X \bar{\Phi}(\cdot), \quad(\cdot) \in Y_{1} .
\end{aligned}
$$

By virtue of the Hahn-Banach theorem, each linear functional $\gamma_{i}$ can be extended to the entire space $\mathbf{B}_{1}$ with preservation of norm, and each linear functional $\bar{\varphi}_{s}$ can be extended to the entire space $\mathbf{B}_{2}$. In this connection, we denote the extension of the operator $J: N(L) \rightarrow Y$ to the entire space $\mathbf{B}_{1}$ by $\overline{\mathcal{P}}_{Y_{1}}$ and the extension of its inverse $J^{-1}$ to the space $\mathbf{B}_{2}$ by $\overline{\mathcal{P}}_{N(L)}$, i.e.,

$$
\begin{gathered}
\overline{\mathcal{P}}_{Y_{1}}(\cdot)=\bar{\Psi} \Gamma(\cdot), \quad(\cdot) \in B_{1}, \\
\overline{\mathcal{P}}_{N(L)}(\cdot)=X \bar{\Phi}(\cdot), \quad(\cdot) \in B_{2} .
\end{gathered}
$$

Using (6), we define the projector $\mathcal{P}_{Y_{1}}: \mathbf{B}_{2} \rightarrow Y_{1} \subset Y$ as follows:

$$
\mathcal{P}_{Y_{1}}(\cdot)=\bar{\Psi} \bar{\Phi}(\cdot)
$$

This operator divides the subspace $Y$ into a direct topological sum of subspaces, namely,

$$
Y=Y_{1} \oplus Y_{2},
$$

where $Y_{2}=\mathcal{P}_{Y_{2}} \mathbf{B}_{2}=\left(\mathcal{P}_{Y}-\mathcal{P}_{Y_{1}}\right) \mathbf{B}_{2}$, and is bounded. 
For the class of normally solvable $n$-normal operators, we prove the following statement, which is an analog of the Schmidt lemma:

Lemma 3. Let $L: \mathbf{B}_{\mathbf{1}} \rightarrow \mathbf{B}_{\mathbf{2}}$ be a linear bounded $n$-normal operator and let the image $R(L)$ have a complement in the space $\mathbf{B}_{2}$. Then the operator $\bar{L}=L+\overline{\mathcal{P}}_{Y_{1}}$ has a bounded left-inverse operator:

$$
\bar{L}_{l_{0}}^{-1}=\left(L+\overline{\mathcal{P}}_{Y_{1}}\right)_{l}^{-1}
$$

The general form of the left-inverse operators $\bar{L}_{l_{0}}^{-1}$ is given by the relation

$$
\bar{L}_{l_{0}}^{-1}=\bar{L}_{l_{0}}^{-1}\left(I_{\mathbf{B}_{2}}-\mathcal{P}_{Y_{2}}\right)
$$

Proof. Let $L$ be an $n$-normal operator. For the operator $\bar{L}$ to be left invertible, it is necessary and sufficient that the following conditions be satisfied [9]:

(a) $\operatorname{ker} \bar{L}=\{0\}$;

(b) the linear manifold $R(\bar{L})$ is a subspace that has a direct complement in $\mathbf{B}_{\mathbf{2}}$.

Let us show that $\operatorname{ker} \bar{L}=\{0\}$. Assume that there exists $x_{0} \neq 0, x_{0} \in \mathbf{B}_{\mathbf{1}}$, such that

$$
\left(L+\overline{\mathcal{P}}_{Y_{1}}\right) x_{0}=L x_{0}+\bar{\Psi} \Gamma\left(x_{0}\right)=0 .
$$

It is obvious that $L x_{0} \in R(L)$. It follows from the definition of $\overline{\mathcal{P}}_{Y_{1}}$ that $\overline{\mathcal{P}}_{Y_{1}} x_{0} \in Y_{1} \subset Y$. Since the subspaces $R(L)$ and $Y$ mutually complement one another to the entire space $\mathbf{B}_{2}$, we have $R(L) \bigcap Y=\{0\}$, i.e., they have only one common element, namely the zero element. Thus, $L x_{0}=0$ and $\overline{\mathcal{P}}_{Y_{1}} x_{0}=0$. This implies that $x_{0} \in N(L)$ and $x_{0} \in N\left(\overline{\mathcal{P}}_{Y_{1}}\right) \subset X$. Since the subspaces $N(L)$ and $X$ also mutually complement one another to the space $\mathbf{B}_{1}$, we have $N(L) \bigcap X=\{0\}$. This yields $x_{0}=0$.

The complementability of the image $R(\bar{L})$ in the space $\mathbf{B}_{2}$ follows from relation (7) and the complementability of the subspace $R(L)$ :

$$
\mathbf{B}_{2}=R(L) \oplus Y_{1} \oplus Y_{2}=R(\bar{L}) \oplus Y_{2}
$$

Therefore, the operator $\bar{L}$ has a left inverse. Since the operator $\bar{L}$ maps the Banach space $\mathbf{B}_{\mathbf{1}}$ bijectively to the subspace $\mathbf{B}_{\mathbf{2}} \ominus Y_{2}$, it follows from the Banach theorem [10] that the operator $\bar{L}_{l}^{-1}$ is bounded. It is known [9, p. 61] that if the projection operator $\mathcal{P}$ possesses the property $R(\mathcal{P})=R(\bar{L})$, then the general form of leftinverse operators admits the representation $\bar{L}_{l_{0}}^{-1} \mathcal{P}$. It follows from (8) that the operator $I_{\mathbf{B}_{2}}-\mathcal{P}_{Y_{2}}$ possesses this property, i.e., $R\left(I_{\mathbf{B}_{2}}-\mathcal{P}_{Y_{2}}\right)=R(\bar{L})$. Therefore, the general representation of left-inverse operators can be rewritten as follows:

$$
\bar{L}_{l_{0}}^{-1}=\bar{L}_{l_{0}}^{-1}\left(I_{\mathbf{B}_{2}}-\mathcal{P}_{Y_{2}}\right)
$$

The lemma is proved.

Remark 1. If $\operatorname{dim} \operatorname{ker} L<\operatorname{dim} \operatorname{ker} L^{*}<\infty$, i.e., $L$ is a Noetherian operator of negative index, then Lemma 3 reduces to Lemma 2.4 in [3, p. 47]. 
Remark 2. If $\operatorname{dim} \operatorname{ker} L=\operatorname{dim} \operatorname{ker} L^{*}=n<\infty$, i.e., $L$ is a Fredholm operator of nonzero index, then Lemma 3 reduces to the Schmidt lemma [2, p. 340].

Now let $L: B_{1} \rightarrow B_{2}$ be a linear bounded $d$-normal operator. In this case, the subspace $N(L)$ is infinitedimensional $(\mu=\infty)$ and the subspace $N\left(L^{*}\right)$ is finite-dimensional $(v<\infty)$. Assume that the space $\mathbf{B}_{1}$ has a basis. Then $N(L)$ also has a basis. Let $\left\{f_{i}\right\}_{i=1}^{\infty} \subset N(L)$ be a complete system of basis elements. The subspace $N\left(L^{*}\right)$ has a finite-dimensional basis $\left\{\varphi_{s}\right\}_{s=1}^{v} \subset N\left(L^{*}\right)$. For the elements $\left\{f_{i}\right\}_{i=1}^{\infty}$ and functionals $\left\{\varphi_{s}\right\}_{s=1}^{v}$, there exist an adjoint biorthogonal system of functionals $\left\{\gamma_{j}\right\}_{j=1}^{\infty} \subset \mathbf{B}_{1}^{*}$ and an adjoint biorthogonal complete system of elements $\left\{\psi_{k}\right\}_{k=1}^{v} \subset \mathbf{B}_{2}$ [7]. Each of the functionals $\left\{\gamma_{j}\right\}_{j=1}^{\infty}$ and $\left\{\varphi_{s}\right\}_{s=1}^{v}$ defined on the subspaces $N(L) \subset \mathbf{B}_{1}$ and $Y \subset \mathbf{B}_{2}$, according to the Hahn-Banach theorem, can be extended to the spaces $\mathbf{B}_{1}$ and $\mathbf{B}_{2}$, respectively, with preservation of norm.

By analogy with (3), let

$$
\begin{gathered}
X=\left(f_{1}, f_{2}, \ldots, f_{s}, \ldots\right), \quad \Gamma(\cdot)=\left(\gamma_{1}(\cdot), \gamma_{2}(\cdot), \ldots, \gamma_{s}(\cdot), \ldots\right)^{T}, \\
\Phi(\cdot)=\left(\varphi_{1}(\cdot), \varphi_{2}(\cdot), \ldots, \varphi_{\nu}(\cdot)\right)^{T}, \quad \Psi=\left(\psi_{1}, \psi_{2}, \ldots, \psi_{\nu}\right)
\end{gathered}
$$

denote $\infty \times \infty, \infty \times \infty, v \times \infty$, and $\infty \times v$ matrices, respectively; furthermore, $\Gamma(X)=E_{\infty}$ and $\Phi(\Psi)=E_{\nu}$, where $E_{\infty}$ and $E_{v}$ are the identity matrices.

To construct a projection operator $\mathcal{P}_{N(L)}: B_{1} \rightarrow N(L)$, we define the sequence of projectors

$$
\mathcal{P}_{N^{(i)}(L)}(\cdot)=X_{i} \Gamma_{i}(\cdot), \quad i=1,2,3, \ldots
$$

of the space $B_{1}$ to the subspaces $N_{i}(L)$ of the null space $N(L)$.

Lemma 4. The sequence (9) of projectors $\mathcal{P}_{N^{(i)}(L)}$ converges strongly (pointwise) to the projector

$$
\mathcal{P}_{N(L)}(\cdot)=X \Gamma(\cdot)=\lim _{i \rightarrow \infty} X_{i} \Gamma_{i}(\cdot), \quad \mathcal{P}_{N(L)}: B_{1} \rightarrow N(L)
$$

Proof. The proof is analogous to the proof of Lemma 1.

We define a projection operator $\mathcal{P}_{Y}: \mathbf{B}_{2} \rightarrow Y$ of the space $\mathbf{B}_{2}$ to the subspace $Y$ as follows:

$$
\mathcal{P}_{Y}(\cdot)=\Psi \Phi(\cdot)
$$

Note that, for the projection operators (10) and (11), Lemma 2 is true.

Since $\mu$ is infinite and $v$ is finite, we can establish an isomorphism between $N_{1}(L) \subset N(L)$ and $Y$.

We now construct this isomorphism. Let

$$
\bar{X}=\left(\bar{f}_{1}, \bar{f}_{2}, \ldots, \bar{f}_{v}\right) \quad \text { and } \quad \bar{\Gamma}(\cdot)=\left(\bar{\gamma}_{1}(\cdot), \bar{\gamma}_{2}(\cdot), \ldots, \bar{\gamma}_{v}(\cdot)\right)^{T}
$$

denote, respectively, $\infty \times v$ and $v \times \infty$ matrices. Then we construct a linear, bounded, invertible operator $J: N_{1}(L) \rightarrow Y$ that realizes an isomorphism of $N_{1}(L)$ onto $Y$ and its inverse $J^{-1}: Y \rightarrow N_{1}(L)$ as follows:

$$
\begin{gathered}
J(\cdot)=\Psi \bar{\Gamma}(\cdot), \quad(\cdot) \in N_{1}(L), \\
J^{-1}(\cdot)=\bar{X} \Phi(\cdot), \quad(\cdot) \in Y .
\end{gathered}
$$


The matrix $\bar{X}$ is composed of $v$ columns of the matrix $X$, and the matrix $\bar{\Gamma}(\cdot)$ is composed of functionals of the matrix $\Gamma(\cdot)$ that satisfy the relation $\bar{\Gamma}(\bar{X})=E_{\nu}$.

Let $\overline{\mathcal{P}}_{Y}$ denote an extension of the operator $J: N(L) \rightarrow Y$ to the entire space $B_{1}$ and let $\overline{\mathcal{P}}_{N_{1}(L)}$ denote an extension of its inverse $J^{-1}$ to the space $B_{2}$, i.e.,

$$
\begin{gathered}
\overline{\mathcal{P}}_{Y}(\cdot)=\Psi \bar{\Gamma}(\cdot), \quad(\cdot) \in B_{1}, \\
\overline{\mathcal{P}}_{N_{1}(L)}(\cdot)=\bar{X} \Phi(\cdot), \quad(\cdot) \in B_{2} .
\end{gathered}
$$

By analogy with (11), we define the projection operator $\mathcal{P}_{N_{1}(L)}: \mathbf{B}_{1} \rightarrow N_{1}(L) \subset N(L)$ as follows:

$$
\mathcal{P}_{N_{1}(L)}(\cdot)=\bar{X} \bar{\Gamma}(\cdot)
$$

This operator is bounded and divides the subspace $N(L)$ into a direct topological sum of subspaces:

$$
N(L)=N_{1}(L) \oplus N_{2}(L), \quad N_{2}(L)=\mathcal{P}_{N_{2}(L)} \mathbf{B}_{1},
$$

where $\mathcal{P}_{N_{2}(L)}=\mathcal{P}_{N(L)}-\mathcal{P}_{N_{1}(L)}$ is a bounded projector.

For the class of normally solvable $d$-normal operators, we prove a statement analogous to the Schmidt lemma.

Lemma 5. Let $L: \mathbf{B}_{1} \rightarrow \mathbf{B}_{\mathbf{2}}$ be a linear bounded $d$-normal operator and let the kernel $N(L)$ have a complement in the space $\mathbf{B}_{1}$. Then the operator $\bar{L}=L+\overline{\mathcal{P}}_{Y}$ has a bounded right-inverse operator:

$$
\bar{L}_{r_{0}}^{-1}=\left(L+\overline{\mathcal{P}}_{Y}\right)_{r}^{-1}
$$

The general form of the right-inverse operators $\bar{L}_{r_{0}}^{-1}$ is given by the relation

$$
\bar{L}_{r_{0}}^{-1}=\left(I_{\mathbf{B}_{1}}-\mathcal{P}_{N_{2}(L)}\right) \bar{L}_{r}^{-1}
$$

Proof. For the operator $\bar{L}$ to be right invertible, it is necessary and sufficient that the following conditions be satisfied [9]:

(a) $R(\bar{L})=\mathbf{B}_{2}$;

(b) the subspace $N(\bar{L})$ has a direct complement in $\mathbf{B}_{\mathbf{1}}$.

Using the second equality in (5), we get $R(L)=N\left(\mathcal{P}_{Y}\right)$, i.e., the condition $R(\bar{L})=\mathbf{B}_{2}$ is equivalent to the condition

$$
\mathcal{P}_{Y}(\cdot)=\Psi \Phi(\cdot)=0 .
$$

Since the system of elements $\left\{\psi_{s}\right\}_{s=1}^{v}$ is linearly independent, the last relation holds if and only if all elements of $\left\{\varphi_{s}\right\}_{s=1}^{v}$ are equal to zero. This, in turn, means that the null space of the adjoint operator is trivial, i.e., $N\left(L^{*}\right)=\{0\}$. 
Let us show that $N\left(\bar{L}^{*}\right)=\{0\}$. Assume that there exists a functional $\varphi_{0}, \varphi_{0} \neq 0, \varphi \in \mathbf{B}_{2}^{*}$, such that $\bar{L}^{*} \varphi_{0}=\left(L+\overline{\mathcal{P}}_{Y}\right)^{*} \varphi_{0}=0$. Taking into account the definition of the operator $\overline{\mathcal{P}}_{Y}$, we get

$$
L^{*} \varphi_{0}=-\overline{\mathcal{P}}_{Y}^{*} \varphi_{0}
$$

Applying the functionals $L^{*} \varphi_{0} \in \mathbf{B}_{1}^{*}$ and $\overline{\mathcal{P}}_{Y}^{*}$ to the matrix $X$, we obtain, on the one hand,

$$
\left(L^{*} \varphi_{0}\right)(X)=\varphi_{0}(L X)=0
$$

because $L X=0$ and, on the other hand,

$$
\overline{\mathcal{P}}_{Y}^{*} \varphi_{0}(X)=\varphi_{0}\left(\overline{\mathcal{P}}_{Y} X\right)=\varphi_{0}(\Psi) \bar{\Gamma}(X)=\varphi_{0}(\Psi)
$$

because $\bar{\Gamma}(X)=\delta_{i j}$. Since the system of elements $\left\{\psi_{i}\right\}_{i=1}^{v}$ is linearly independent, the equality $\varphi_{0}(\Psi)=0$ is possible only for $\varphi_{0}=0$. This contradiction proves that $N\left(\bar{L}^{*}\right)=\{0\}$, which, in turn, means that $R(\bar{L})=\mathbf{B}_{2}$.

The complementability of the null space $N(\bar{L})$ follows from the definition of the projector $\mathcal{P}_{N_{1}(L)}(13)$ and the decomposition (14) of the null space $N(L)$ of the operator $L$.

It is known [9, p. 62] that if a projection operator $\mathcal{P}$ possesses the property $N(\mathcal{P})=N(\bar{L})$, then the general form of right-inverse operators admits the representation $\mathcal{P} \bar{L}_{r_{0}}^{-1}$. It follows from (14) that the operator $I_{\mathbf{B}_{1}}-$ $\mathcal{P}_{N_{2}(L)}$ possesses this property, i.e., $N\left(I_{\mathbf{B}_{1}}-\mathcal{P}_{N_{2}(L)}\right)=N(\bar{L})$. Therefore, the general representation of rightinverse operators can be rewritten as follows:

$$
\bar{L}_{r_{0}}^{-1}=\left(I_{\mathbf{B}_{1}}-\mathcal{P}_{N_{2}(L)}\right) \bar{L}_{r}^{-1}
$$

The lemma is proved.

Remark 3. If $\operatorname{dim} \operatorname{ker} L^{*}<\operatorname{dim} \operatorname{ker} L<\infty$, i.e., $L$ is a Noetherian operator of positive index, then Lemma 5 reduces to Lemma 2.4 in [3, p. 47].

\section{REFERENCES}

1. E. Schmidt, "Zur Theorie linearen und nichtlinearen Integralgleichungen. Teil 3. Über die Auflösungen der nichtlinearen Integralgleichungen und die Verzweigung ihrer Losungen," Math. Ann., No. 65 (1908).

2. M. M. Vainberg and V. A. Trenogin, Theory of Branching of Solutions of Nonlinear Equations [in Russian], Nauka, Moscow (1969).

3. A. A. Boichuk, V. F. Zhuravlev, and A. M. Samoilenko, Generalized Inverse Operators and Noetherian Boundary-Value Problems [in Russian], Institute of Mathematics, Ukrainian National Academy of Sciences, Kiev (1995).

4. S. G. Krein, Linear Differential Equations in a Banach Space [in Russian], Nauka, Moscow (1967).

5. Yu. L. Daletskii and M. G. Krein, Stability of Solutions of Differential Equations in a Banach Space [in Russian], Nauka, Moscow (1970).

6. L. A. Lyusternik and V. I. Sobolev, A Brief Course in Functional Analysis [in Russian], Vysshaya Shkola, Moscow (1982).

7. M. M. Grinblyum, "Biorthogonal systems in a Banach space," Dokl. Akad. Nauk SSSR, 47, No. 2, 79-82 (1945).

8. M. I. Kadets and B. S. Mityagin, "Complementable subspaces in Banach spaces," Usp. Mat. Nauk, 28, Issue 6, 77-94 (1973).

9. I. Ts. Gokhberg and N. Ya. Krupnik, Introduction to the Theory of One-Dimensional Singular Integral Operators [in Russian], Shtiintsa, Kishinev (1973).

10. V. A. Trenogin, Functional Analysis [in Russian], Nauka, Moscow (1989). 